\title{
Patient Safety Climate: professionals sense in a rheumatology clinic
}

Carolina Teixeira Cunha (Universidade Nova de Lisboa, Portugal), Fabiana Miranda Moura Santos (Pulsus Inovação em Saúde, Belo Horizonte, MG, Brasil), Cláudia Tártaglia Reis (Universidade Nova de Lisboa, Portugal)

\section{BACKGROUND}

Climate measure of patient safety is a validated practice and allows identification of potentialities and weaknesses of assistance, as well as planning of future interventions. Objective: To evaluate the sense of the safety climate among the professionals working in a private rheumatology clinic in Belo Horizonte (MG).

\section{MATERIALS AND METHODS}

A cross-sectional, descriptive study using the Safety Attitudes Questionnaire (SAQ) Short form 2006, translated and validated in portuguese. Composed of sociodemographic data and 41 items that cover six domains: 1) Team climate, 2) Safety climate, 3) Work satisfaction, 4) Stress perception, 5) Management perception, 6) Working conditions. The answers to each of the questions follow a Likert scale and a score: I totally disagree $(A)=0$, slightly disagree $(B)=25$, neutral $(C)=50$, agree a little $(D)=75, I$ totally agree, $(E)=100$ and does not apply 0 . The final score of the instrument varies from 0 to 100, where 0 represents the worst perception of the safety climate and 100 the best. The descriptive analysis of the questionnaire was performed by the means and standard deviation (DP) of the responses to items ( 1 to 5 ) after inversion of the reverse items and by the calculation of the professionals' responses to the 41 items. Values greater than 75 signify strong agreement of the professionals regarding the questions. Of the 27 participating professionals, 4 were excluded because they did not meet the eligibility criteria and 3 were excluded because they did not meet the quality control of the data.

\section{RESULTS}

The study population consisted of 20 professionals, $40 \%$ (doctors), 20\% (nursing staff), 25\% (administrative) and 15\% (management). Of these, 35\% worked as professionals for 4 years and $65 \%$ have been working for more than 4 years. The descriptive analysis of the domains pointed to the average of the following scores: Teamwork Climate 89.0 (SD:0.29), Safety Climate 87.25 (SD:0.29), Job Satisfaction 96.75 (SD:0.08), Stress Perception 70 (SD:0.47), Management Perception 83.25 (SD:0.29), Work Design 86.5. A positive sense of the safety climate was evidenced by total score with a mean of 85.89 (SD:10.76).

\section{CONCLUSION}

This work showed a professional satisfaction and a good teamwork climate, which contributes directly to the patient's effective safety culture. Only the domain Stress Perception presented a negative score indicating the need for planning and development of targeted strategies. 